\title{
Gestión de calidad como estrategia clave de la felicidad en el deporte federado
}

\section{Quality Management as a key strategy for happiness in federation-based sport}

\begin{abstract}
Cristina Loranca Valle es doctoranda en Comercialización e Investigación de Mercados de la Universidad de Alcalá (España) (cristina.loranca@uah.es) (https://orcid.org/0000-0002-1561-1221)
\end{abstract}

Dr. Pedro Cuesta-Valiño es profesor/investigador de Comercialización e Investigación de Mercados de la Universidad de Alcalá (España) (pedro.cuesta@uah.es) (https://orcid.org/0000-0001-9521-333X)

Dra. Estela Núñez-Barriopedro es vicedecana de calidad, promoción y comunicación, profesora/investigadora de Comercialización e Investigación de Mercados de la Universidad de Alcalá (España) (estela.nunezb@uah.es) (http://orcid.org/0000-00022292-8147)

\section{Resumen}

La relación entre el deporte y la felicidad es un hecho probado en multitud de ocasiones. El ejercicio físico aporta al deportista diversas sensaciones positivas, todas ellas muy relacionadas con el término felicidad. Por tanto, partiendo del hecho de que la práctica deportiva produce felicidad, el objetivo de este estudio es de averiguar qué variables influyen principalmente en la felicidad del deportista y en concreto, del deportista federado. Una de las novedades de este estudio es que los resultados podrían ayudar a los miembros de las juntas directivas de las federaciones a tomar buenas decisiones y poder contribuir en mayor forma a la felicidad del deportista federado. Las principales variables que encontramos en la literatura que influyen positivamente en la felicidad son la calidad percibida y la satisfacción. A su vez encontramos estudios que confirman la influencia de la confianza y las relaciones sociales sobre la felicidad. El estudio empírico se ha realizado a través de datos primarios mediante encuesta sobre una muestra de 601 individuos federados en las distintas federaciones autonómicas de España, concretamente en la modalidad deportiva de kárate. Los resultados obtenidos confirman todas las hipótesis planteadas en este estudio por lo que como indicaba la literatura revisada las cuatro variables analizadas (la calidad percibida, la satisfacción, la confianza y las relaciones sociales) influyen de forma significativa y positivamente en la felicidad.

\begin{abstract}
The relationship between sport and happiness has been proven on many occasions. On this way, physical sport gives sportspeople positive feelings which are all related to the term happiness. So, based on the fact that taking part in sports increases happiness, the main objective of this study is therefore to determine which variables have the greatest influence on sportspeople's happiness, focusing specifically on federation members. One of the new developments in this study is that the results may help members of the Governing Boards of these federations to take better decisions and contribute more to the happiness of sportspeople who are federation members. The principal variables mentioned in the literature as having a positive influence on happiness are perceived quality and satisfaction. We have also found studies that confirm the influence of both trust and social relationships on happiness. The empirical research has used primary data from a survey of 601 members of the Spanish regional federations, specifically in the sport modality of karate. Our results confirm all the hypotheses advances in this study. Thus, as indicated by the literature reviewed, the four variables studied (perceived quality and satisfaction, trust and social relationships) all exert significant positive influence on levels of happiness.
\end{abstract}

\section{Palabras clave I keywords}

Felicidad, satisfacción, calidad percibida, confianza, deporte, kárate, federaciones, relaciones sociales. Happiness, satisfaction, perceived quality, trust, sport, karate, federations, social relationships.

Cómo citar: Loranca Valle, C., Cuesta-Valiño, P. y Núñez-Barriopedro, E. (2019). Gestión de calidad como estrategia clave de la felicidad en el deporte federado. Retos Revista de Ciencias de la Administración y Economía, 9(18), 203-218. https://doi.org/10.17163/ret.n18.2019.02 


\section{Introducción}

La felicidad es algo que han estado buscando las personas desde el principio de los tiempos. La consecución de los objetivos es una de las razones por las cuales las personas experimentan la felicidad (Núñez-Barriopedro, Ruiz-López, \& Ravina Ripoll, 2018). En este sentido, conseguir objetivos deportivos puede ayudar alcanzar la felicidad, por lo que se deduce que el deporte y la felicidad están estrechamente vinculados. La práctica deportiva está asociada a la tenencia de salud. De hecho, la falta de ejercicio físico y el sedentarismo se han vinculado con algunas enfermedades y trastornos (Álvarez, 2007). Ésta es una de las razones por la que los gobiernos promueven el deporte: mejorar la salud, luchar contra la obesidad, frenar el crimen, y proporcionar valores a la juventud (Huang \& Humphreys, 2012; Hur, Ko, \& Valacich, 2011). Concretamente en España [...] "el desarrollo del Deporte de alto nivel y alto rendimiento reside en las Federaciones Deportivas Españolas con las colaboraciones de las Comunidades Autónomas. Está financiado principalmente por el Estado y su objetivo es elevar el nivel deportivo de España internacionalmente" (Consejo Superior de Deportes, 2019, s/p).

El objeto de ser de una federación deportiva es el de promover e impulsar el deporte (Supreme Council for Sports in Spain. Ministry of Education, Culture and Sports, 2007), y aunque son organizaciones privadas, la Administración Pública es quien las dota como órgano para el desarrollo del deporte de alto nivel y alto rendimiento. Así pues, la Administración aporta las principales dotaciones económicas a estas organizaciones con el objetivo de elevar el nivel deportivo de España internacionalmente (Supreme Council for Sports in Spain. Ministry of Education, Culture and Sports, 2007).

Algunas investigaciones han demostrado que el consumo deportivo como espectador (Hallmann, Breuer \& Ku, 2013; Jang, Ko, Wann \& Kim, 2017) las vacaciones deportivas, la asistencia a eventos deportivos (Nicolao, Irwin \& Goodman, 2009), o la participación en eventos (Huang \& Humphreys, 2012; Theodorakis, Kaplanidou \& Karabaxoglou, 2015) están muy vinculados a la felicidad y aumentan los niveles de ésta.

A pesar del interés creciente de la población en la actividad física y el deporte, no se percibe un crecimiento proporcional de miembros de organizaciones deportivas sin ánimo de lucro (Wemmer \& Koenigstorfer, 2016). Aunque durante los últimos 10 años el número de licencias federadas se ha mantenido en una cifra constante, sí que se aprecia un crecimiento en los últimos 2 años, pero si ampliamos la línea temporal la variación es apenas perceptible. Las organizaciones deportivas lucrativas han entrado en el mercado con mucha fuerza y están atrayendo más y más consumidores. Sin embargo, sus pares no lucrativos no les están plantando cara a este incremento de la competencia (Smith \& Stewart, 2010).

En este contexto, el presente trabajo trata de estudiar las causas de que apenas se produzca variación en las licencias a lo largo de estos últimos 10 años. Si bien es cierto que las federaciones son organizaciones sin ánimo de lucro, su razón de ser es la promoción y divulgación del deporte y esto conlleva a que uno de sus principales intereses sea hacer felices a sus federados a través del deporte, por lo que el presente 
trabajo puede aportar conocimiento especialmente para los miembros de las juntas directivas de las federaciones con el objetivo de conocer cuáles son las variables que pueden influir en la felicidad de sus federados para que tengan la oportunidad de gestionarlas de la mejor manera posible y así conseguir su propósito y misión.

\subsection{Estado de la cuestión}

\subsubsection{Felicidad}

La felicidad aporta una serie de sensaciones, las cuales cualquier persona mentalmente sana quisiera mantener el máximo tiempo posible (Ravina-Ripoll et al., 2019). Algunos autores definen la felicidad como el estado psicológico positivo derivado de una experiencia buena, agradable y satisfactoria (Bolifa et al., 2017; Jang et al., 2017). Existen dos perspectivas desde las que se puede analizar la felicidad: una alude a un momento concreto en el tiempo y la otra a una duración constante. La primera, es la consecuencia de una situación o experiencia positiva particular, mientras que la segunda es un estado psicológico positivo general que es acumulativo en el tiempo (Seligman \& Csikszentmihalyi, 2014).

De acuerdo a numerosos estudios, la felicidad y la lealtad están positivamente relacionadas. Aksoy et al. (2015) confirma empíricamente que tanto la lealtad concreta como la lealtad abstracta influyen en la felicidad. De hecho, el óptimo balance entre una y otra contribuirán a maximizar la felicidad general. Que el cliente esté satisfecho no implica que sea feliz, pero para llevar al cliente a la lealtad hay que buscar su felicidad (Khan \& Hussain, 2013) más que concentrarse en su satisfacción, que es lo que se ha estado persiguiendo durante los últimos 50 años (Easterlin, 2001).

La calidad de vida y la felicidad se ha vinculado en multitud de ocasiones, y no solo eso, sino que cuando una gran parte de la población es feliz, implicará que la calidad de vida de esa población es buena (Barriopedro, Ripoll, \& Tello, 2018a). Las personas felices tienden a tener vidas prósperas, tanto de forma externa como interna (Nelson et al., 2015). En la literatura sobre felicidad, algunos investigadores sostienen que la felicidad de una determinada persona dependerá de una serie de factores relacionados con sentimientos positivos, como son las relaciones sociales, el trabajo y el desempleo, el ocio, el dinero, la clase, la cultura, la personalidad, la alegría, la satisfacción con la vida, edad, sexo, salud, entre otras. Todo esto quiere decir que las circunstancias generales de la vida repercuten en la felicidad -como constructo constante- (v. gr. Argyle, 1994; Barriopedro, Ripoll, \& Tello, 2018b; Ravina-Ripoll et al., 2019).

\subsubsection{Calidad}

La definición de calidad percibida más defendida por los autores se refiere a las discrepancias entre las percepciones del consumidor sobre un determinado bien o servicio ofrecido y las expectativas sobre dicho servicio (v.gr. Hennig-Thurau, Gwinner, \& Gremler, 2002; Cuesta Valiño, Gutiérrez Rodríguez, \& Núñez Barriopedro, 2019). En igualdad de percepciones, cuanto mayores sean las expectativas del consumidor, menor será la calidad percibida. La calidad percibida es el juicio del consumidor sobre la excelencia o superioridad general de una entidad y difiere de la calidad ob- 
jetiva. El significado conceptual de calidad tiene dos dimensiones: una mecánica y otra humanística. La dimensión mecánica se refiere a las características objetivas de la transacción, mientras que la dimensión humanística de la calidad es relativa a la respuesta subjetiva de la gente frente a un determinado caso y depende del juicio de cada persona (Gallarza, et al., 2017).

Para el caso concreto de las federaciones deportivas, como el producto ofertado son servicios, los clientes perciben la relación de intercambio con un mayor riesgo. Esto es debido a las principales características de los servicios: intangibilidad y heterogeneidad. Por eso la credibilidad es uno de los atributos que mayor importancia tiene para el consumidor a la hora de evaluar la calidad percibida (Javalgi \& Moberg, 1997).

\subsubsection{Satisfacción}

La satisfacción se puede conceptualizar de dos formas: la satisfacción de una transacción específica y la satisfacción acumulada (Boulding et al., 1993), siendo esta última la evaluación general basada en la totalidad de las compras y de las experiencias de consumo de un determinado bien o servicio (Anderson \& Mansi, 2009).

Otros autores la definen como la reacción emocional o sentimental del cliente a las diferencias percibidas entre la ejecución del trabajo y las expectativas. Aunque esta definición podría llevar a confusión por sus similitudes con la definición de «calidad percibida», lo cierto es que ambas variables son diferentes residiendo la principal diferencia en que la satisfacción es un tipo de actitud, una evaluación a largo plazo, sin embargo, la calidad percibida es la medida de una transacción específica. Núñez-Barriopedro, RuizLópez, \& Ravina Ripoll (2018) relacionan la satisfacción con el hecho de proporcionar lo que se está buscando hasta el punto de que se pueda alcanzar ésta.

\subsubsection{Confianza}

La confianza es uno de los ingredientes básicos para tener éxito en las relaciones (Cuesta Valiño, Gutiérrez Rodríguez, \& Núñez Barriopedro, 2019), siendo ésta la creencia de una de las partes de que las acciones que la otra parte ejecuta necesariamente le satisfarán (Anderson \& Mansi, 2009).

Por su parte, la confianza en una organización viene dada por la seguridad del consumidor en la calidad y la integridad del servicio ofrecido (Hennig-Thurau, Langer, \& Hansen, 2001). En la confianza de las relaciones entre el consumidor y las compañías tiene más relevancia el beneficio psicológico de la seguridad y la confianza que el trato especial o los beneficios sociales derivados de esta relación (Eaton, Gwinner, Larson \& Swanson, 2015). La confianza es la implicación en un proceso que ha sido bien pensado y cuidadosamente considerado, mientras que el afecto a una marca es más bien espontaneo, más inmediato y menos razonado. En este sentido, la confianza puede tener una dimensión afectiva y otra cognitiva (Johnson \& Grayson, 2005).

\section{Relación entre calidad percibida y felicidad}

Mejorar la calidad del servicio en la compra de eventos deportivos es una oportunidad para mejorar de forma positiva la psicología del consumidor (Anderson \& Mansi, 
2009). En la literatura podemos encontrar diversos autores que demuestran y confirman la relación existente entre calidad percibida o calidad del servicio percibida y la felicidad (v.gr. Gong \& Yi, 2018; Sato, Jordan \& Funk, 2014; Theodorakis et al., 2015).

En su estudio, Theodorakis et al. (2015) tratan de averiguar la relación existente y la influencia de la calidad percibida y la satisfacción sobre la felicidad que puede producir el consumo de eventos deportivos. Para ello se basan en las afirmaciones de Sato, Jordan y Funk (2014), quienes encontraron que "el ocio físicamente activo puede mejorar la calidad de vida de los participantes al proporcionar experiencias positivas a través de la participación en eventos" (p. 298). En lo que se refiere a los eventos deportivos, Theodorakis et al. (2015, op. cit.) diferencian tres tipos de calidad del servicio: la calidad de los resultados, la calidad de interacción y la calidad del entorno físico, siendo únicamente la variable de calidad de resultados la que influye sobre la felicidad.

La satisfacción es una variable muy relacionada también con la calidad percibida. En ocasiones actúa como variable mediadora entre ésta y la felicidad, como ocurre en el estudio de Gong y Yi (2018), en el cual miden varios aspectos de las percepciones sobre la calidad general del servicio como puede ser el ambiente, el desarrollo o la entrega de dicho servicio. Estas tres variables influyen sobre la satisfacción que a su vez influye sobre la lealtad y la felicidad.

Teniendo en cuenta las aportaciones de la literatura revisada sobre las relaciones ente calidad percibida y felicidad, se platean las siguientes hipótesis:

H1: La calidad percibida influye positivamente sobre la satisfacción

H2: La calidad percibida influye positivamente sobre la felicidad

\subsubsection{Relación entre satisfacción y felicidad}

Mejorar la calidad del servicio en la compra de eventos deportivos es una oportunidad para mejorar de forma positiva la psicología del consumidor (Anderson \& Mansi, 2009).

La felicidad y la satisfacción con la vida son dos conceptos diferentes, pero en su investigación Haller y Hadler (2006) encuentran que los factores macrosociales, como la riqueza económica de una nación, la distribución de las rentas, la extensión del estado del bienestar, y la libertad política influyen tanto en la felicidad como en la satisfacción. En esta línea, cuando un servicio se produce repetidas veces en el tiempo, las percepciones derivadas de estos encuentros forman la base de la satisfacción del consumidor y a su vez, esto conduce a la felicidad del consumidor (Dagger \& Sweeney, 2006).

Por otro lado, la satisfacción del cliente se extiende a cualquier momento en la vida, lo que conduce a la felicidad (Sweeney, Danaher \& Mccoll-Kennedy, 2015). Esto podría asimilarse con la teoría de abajo hacia arriba de la felicidad del cliente, la cual establece que la satisfacción del cliente, derivada de un servicio específico, se extiende hacia arriba a la satisfacción general del servicio, y ésta, a su vez se extiende hacia la felicidad (Gong \& Yi, 2018). Las conclusiones obtenidas de los trabajos analizados sobre satisfacción y felicidad nos hacen plantear la siguiente hipótesis:

H3: La satisfacción influye positivamente sobre la felicidad. 


\subsubsection{Relación entre confianza y felicidad}

Tal como se ha apuntado ut supra, mejorar la calidad del servicio en la compra de eventos deportivos es una oportunidad para mejorar de forma positiva la psicología del consumidor (Núñez-Barriopedro, Ravina Ripoll \& Tobar Pesantez, 2019). La literatura que analiza la relación entre la confianza y la felicidad se ha estudiado principalmente con datos agregados a escala de región o nacional (Barra, Pressgrove \& Torres, 2018; Bartolini \& Mikucka, 2017; Tokuda, Fujii \& Inoguchi, 2010), buscando la perspectiva geográfica.

En sus investigaciones Bartolini y Mikucka (2017) analizan la relación existente entre la confianza social y el bienestar subjetivo o la felicidad en el este de Europa. Para ellos la confianza no influye sobre la felicidad en el corto plazo, sin embargo, en el medio-largo plazo, la influencia de la confianza sobre la felicidad se equipara a la influencia del crecimiento económico sobre la felicidad. En otro estudio sobre la relación entre la confianza y la felicidad en el este de Japón, se defiende que la relación entre ambas variables depende del contexto en el que se analice (Barra et al., 2018).

La confianza puede jugar un papel muy importante en la reducción de posibles influencias negativas en la felicidad que pueden llegar a surgir cuando las leyes no funcionan bien (Barra et al., 2018). Las personas que viven en países con mayores niveles de confianza social agregada tienen más probabilidades de ser felices que las que viven en países con un nivel de confianza más pobre (Tokuda et al., 2010).

Basándonos en las líneas de pensamiento de la literatura revisada sobre confianza y felicidad, surge la siguiente hipótesis:

H4: La confianza influye positivamente sobre la felicidad

\subsubsection{Relación entre relaciones sociales y felicidad}

La felicidad y la satisfacción no pueden obtenerse si se dejan de lado las relaciones sociales o la sociedad (Haller \& Hadler, 2006). Las personas siempre han pensado en la estructura y las instituciones sociales en vista de la posibilidad de conseguir una larga vida y felicidad (Boudon, 2002). Haller y Hadler (2006) en su estudio demuestran su hipótesis sobre que ciertas relaciones sociales en determinadas condiciones pueden producir felicidad o lo contrario, infelicidad.

En algunos estudios deportivos se demuestra que las relaciones sociales influyen en la felicidad del espectador deportivo a través de la identificación con el equipo como variable mediadora: la relaciones con otros fans potencian la identificación con el equipo y ésta a su vez influye sobre la felicidad (Jang et al., 2017), ya que los individuos experimentan una mayor felicidad al participar en actividades que facilitan conexiones sociales positivas y de alta calidad con otros (Haller \& Hadler, 2006; Jang et al., 2017). Existen diversos autores que coinciden en que las relaciones sociales y la felicidad están íntimamente relacionadas (RomeroRodríguez \& Castillo-Abdul, 2019).

Algunos autores que investigan cómo influye la amistad en la felicidad, analizan varios aspectos de la amistad como pueden ser la calidad y los conflictos (v.gr. Demir \& Urberg, 2004; Demir \& Weitekamp, 2007). Demir y Weitekamp (2007) demuestran empíricamente que estos dos aspectos de la amistad influyen en la felici- 
dad atendiendo a otras dos variables: el género y la personalidad. Teniendo en cuenta estas líneas de pensamiento, se plantea la siguiente hipótesis:

H5: Las relaciones sociales influyen positivamente sobre la felicidad que produce hacer ejercicio.

\section{Materiales y método}

\subsection{Diseño de la muestra}

El estudio se ha desarrollado sobre la población de federados deportivos españoles. Las federaciones deportivas en España son organizaciones que tienen un carácter muy especial ya que son organizaciones privadas sin ánimo de lucro, aunque son colaboradoras de la Administración Pública y en muchas ocasiones, ejercen como agentes de ésta, llevando a cabo funciones propias de la Administración Pública. Las federaciones deportivas son organizaciones mixtas, teniendo carácter privado y público a la vez. El principal propósito de una federación deportiva es el de promover e impulsar el deporte y aunque son organizaciones privadas, la Administración Pública es quien las dota como órgano para el desarrollo del deporte de alto nivel y alto rendimiento. En este sentido, la Administración aporta las principales dotaciones económicas a estas organizaciones con el objetivo de elevar el nivel deportivo de España internacionalmente. En España existen un total de 66 federaciones, que sumaron en total 3.761.498 licencias deportivas en 2017, mientras que para 2008 el número de licencias federadas alcanzó los 3.394.384 individuos. Podemos observar que sí se ha producido un incremento en estos diez años (+367.114), sin embargo, la línea de crecimiento se ha sostenido constante en el tiempo sobre todo considerando la variación en población en España.

La información recogida se ha obtenido de una muestra de 601 individuos de los que el $71 \%$ son hombres y el $29 \%$ mujeres, todos ellos federados de la modalidad deportiva de kárate. En la muestra encontramos individuos de todas las edades, siendo el grupo más relevante el que tiene el rango de edad entre los 45 y los 64 años, y además la mayoría llevan más de 20 años federados. Se obtuvieron respuestas de 11 federaciones autonómicas, de un total de 19 existentes.

A través de Google Forms, fue enviado el cuestionario autoadministrado a varias federaciones autonómicas españolas de kárate para que los propios federados se lo pasaran a otros federados, a través de un enlace, utilizando la técnica de bola de nieve. Los federados rellenaron la encuesta durante los meses de marzo y abril de 2019.

\section{Análisis y resultados}

De acuerdo a la prueba de ji cuadrado con 16 grados de libertad practicada sobre la muestra, se confirma para todos los indicadores de la variable calidad percibida su relación con la felicidad. Además, en la tabla 1 podemos observar los resultados de la prueba F de Snedecor, cuyos resultados nos confirma que existen diferencias significativas entre los diferentes grupos, ya que la $\mathrm{p}$ valor $<0.05$. 


\section{Tabla 1. Valores medios de los indicadores de calidad percibida según el grado de felicidad de los federados}

\begin{tabular}{|c|c|c|c|c|c|c|c|}
\hline \multirow[b]{2}{*}{ Denominación } & \multirow[b]{2}{*}{$\begin{array}{c}\text { Total } \\
\text { muestra }\end{array}$} & \multicolumn{5}{|c|}{$\begin{array}{l}\text { Participar en las actividades de mi } \\
\text { federación me hace feliz }\end{array}$} & \multirow[t]{2}{*}{$\begin{array}{c}\text { F de } \\
\text { Snedecor }\end{array}$} \\
\hline & & 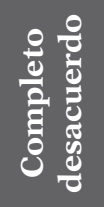 & 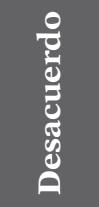 & $\frac{\pi}{\frac{\pi}{0}}$ & \begin{tabular}{l}
0 \\
\multirow{0}{0}{} \\
0 \\
0 \\
0 \\
0 \\
0 \\
0
\end{tabular} & 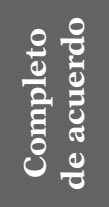 & \\
\hline \multirow{2}{*}{$\begin{array}{l}\text { Las actividades } \\
\text { de mi federación } \\
\text { (campeonatos, cur- } \\
\text { sos, entrenamientos, } \\
\text { etc.) se desarrollan } \\
\text { de manera eficiente. }\end{array}$} & 3.8854 & 1.9556 & 2.9149 & 3.4919 & 4.1392 & 4.5044 & $\begin{array}{c}\mathrm{F}(4.597)= \\
100.8089\end{array}$ \\
\hline & $n=602$ & $\mathrm{n}=45$ & $\mathrm{n}=47$ & $\mathrm{n}=124$ & $\mathrm{n}=158$ & $\mathrm{n}=228$ & $\mathrm{p}=0.0000$ \\
\hline \multirow[t]{2}{*}{$\begin{array}{l}\text { Mi federación } \\
\text { proporciona un } \\
\text { ambiente agrada- } \\
\text { ble para realizar } \\
\text { las actividades que } \\
\text { organiza. }\end{array}$} & 3.9717 & 1.8444 & 2.8723 & 3.6129 & 4.2468 & 4.6256 & $\begin{array}{c}F(4.596)= \\
141.7542\end{array}$ \\
\hline & $n=601$ & $\mathrm{n}=45$ & $\mathrm{n}=47$ & $\mathrm{n}=124$ & $\mathrm{n}=158$ & $\mathrm{n}=227$ & $\mathrm{p}=0.0000$ \\
\hline \multirow[t]{2}{*}{$\begin{array}{l}\text { Entiendo que mi } \\
\text { federación cobra un } \\
\text { precio justo por las } \\
\text { actividades en las } \\
\text { que yo participo. }\end{array}$} & 3.9214 & 2.2045 & 3 & 3.3852 & 4.1329 & 4.5859 & $\begin{array}{c}\mathrm{F}(4.593)= \\
82.3918\end{array}$ \\
\hline & $\mathrm{n}=598$ & $\mathrm{n}=44$ & $\mathrm{n}=47$ & $\mathrm{n}=122$ & $\mathrm{n}=158$ & $\mathrm{n}=227$ & $\mathrm{p}=0.0000$ \\
\hline \multirow[t]{2}{*}{$\begin{array}{l}\text { Los empleados de } \\
\text { mi federación me } \\
\text { atienden de forma } \\
\text { eficaz mis consultas. }\end{array}$} & 4.0266 & 2.2889 & 3.0851 & 3.5691 & 4.2025 & 4.6886 & $\begin{array}{c}\mathrm{F}(4.596)= \\
93.4056\end{array}$ \\
\hline & $\mathrm{n}=601$ & $\mathrm{n}=45$ & $\mathrm{n}=47$ & $\mathrm{n}=123$ & $\mathrm{n}=158$ & $n=228$ & $\mathrm{p}=0.0000$ \\
\hline
\end{tabular}

Además, se ha llevado a cabo una prueba para analizar la varianza unidireccional entre la felicidad y la calidad percibida confirmándose nuestro al planteamiento al rechazarse la hipótesis de independencia de las variables, ya que el resultado obtenido de la F de Snedecor para 4 y 594 grados de libertad fue de 155.2908, con un porcentaje de varianza explicada superior al 50\%, en concreto un $51.12 \%$. En la tabla 2 podemos observar la suma de cuadrados para las variables «Participar en las actividades de mi federación me hace feliz» $\mathrm{y}$ «Los servicios y actividades que ofrece mi federación en global los considero de calidad»: 
Tabla 2. Análisis de la varianza de los indicadores «Participar en las actividades de mi federación me hace feliz« $y$ «Los servicios y actividades que ofrece mi federación en global los considero de calidad»

\begin{tabular}{|c|c|c|c|c|}
\hline Grupos & $\begin{array}{l}\mathbf{N}^{0} \text { de } \\
\text { casos }\end{array}$ & $\begin{array}{c}\text { Media } \\
\text { aritmética }\end{array}$ & $\begin{array}{c}\text { Desviación } \\
\text { estándar }\end{array}$ & $\begin{array}{l}\text { Suma de } \\
\text { cuadrados }\end{array}$ \\
\hline Total muestra & 599 & 3.7963 & 1.232 & 909.1519 \\
\hline \multicolumn{5}{|c|}{ Categorías variable tratamiento } \\
\hline Completo desacuerdo & 26 & 1.5 & 0.9707 & 24.5 \\
\hline Desacuerdo & 31 & 2.0968 & 1.2009 & 44.7097 \\
\hline Indiferencia & 115 & 2.9826 & 1.1032 & 139.9652 \\
\hline De acuerdo & 178 & 3.8258 & 0.7989 & 113.6011 \\
\hline Completo de acuerdo & 249 & 4.6024 & 0.6989 & 121.6386 \\
\hline & & & Suma: & 444.4146 \\
\hline
\end{tabular}

También se puede colegir que existe relación entre todos los indicadores de calidad percibida y los de la satisfacción, puesto que en todas las pruebas de la ji cuadrado la probabilidad resultó ser 0.000. E igualmente la prueba de la F de Snedecor nos indica que existen diferencias significativas en los valores medios de las diferentes tabulaciones cruzadas entre los indicadores de calidad percibida y satisfacción.

Ambas pruebas, ji cuadrado con 16 grados de libertad y F de Snedecor se han realizado para medir la relación entre la satisfacción del federado y la felicidad de estos. La primera prueba confirma que existe relación entre ambas variables, y la segunda que existen diferencias significativas entre los valores medios de los diferentes grupos, como se puede observar en la tabla 3:

\section{Tabla 3. Valores medios de los indicadores de satisfacción según el grado de felicidad de los federados}

\begin{tabular}{|c|c|c|c|c|c|c|c|}
\hline \multirow[b]{2}{*}{ Denominación } & \multirow[b]{2}{*}{$\begin{array}{c}\text { Total } \\
\text { muestra }\end{array}$} & \multicolumn{5}{|c|}{$\begin{array}{l}\text { Participar en las actividades de mi } \\
\text { federación me hace feliz }\end{array}$} & \multirow[b]{2}{*}{$\begin{array}{c}\text { F de } \\
\text { Snedecor }\end{array}$} \\
\hline & & 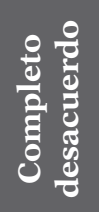 & $\begin{array}{l}0 \\
\frac{0}{0} \\
0 \\
0 \\
0 \\
8 \\
8\end{array}$ & $\begin{array}{l}\frac{9}{0} \\
\frac{\pi}{0} \\
\frac{0}{0} \\
\frac{0}{0}\end{array}$ & \begin{tabular}{l}
0 \\
\multirow{0}{0}{} \\
0 \\
0 \\
0 \\
0 \\
0 \\
0 \\
0
\end{tabular} & 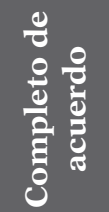 & \\
\hline \multirow[t]{2}{*}{$\begin{array}{l}\text { Creo que es bueno } \\
\text { para mi estar fed- } \\
\text { erado. }\end{array}$} & 4.5717 & 3.3556 & 4.1277 & 4.3468 & 4.6815 & 4.9515 & $\begin{array}{l}\mathrm{F}(4.595)= \\
61.9795\end{array}$ \\
\hline & $\mathrm{n}=600$ & $\mathrm{n}=45$ & $\mathrm{n}=47$ & $\mathrm{n}=124$ & $\mathrm{n}=157$ & $\mathrm{n}=227$ & $\mathrm{p}=0.0000$ \\
\hline
\end{tabular}




\begin{tabular}{|c|c|c|c|c|c|c|c|}
\hline \multirow[b]{2}{*}{ Denominación } & \multirow[b]{2}{*}{$\begin{array}{c}\text { Total } \\
\text { muestra }\end{array}$} & \multicolumn{5}{|c|}{$\begin{array}{c}\text { Participar en las actividades de mi } \\
\text { federación me hace feliz }\end{array}$} & \multirow[b]{2}{*}{$\begin{array}{c}\text { F de } \\
\text { Snedecor }\end{array}$} \\
\hline & & 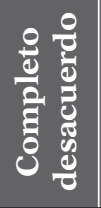 & 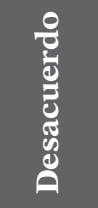 & 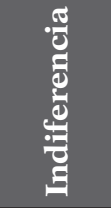 & 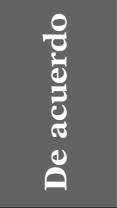 & $\begin{array}{l}\circ \frac{0}{0} \\
\frac{0}{0} \\
\overline{0} \\
\overline{0} \\
0 \\
0 \\
0 \\
0\end{array}$ & \\
\hline \multirow[t]{2}{*}{$\begin{array}{l}\text { Estoy contento de } \\
\text { estar federado en } \\
\text { mi federación. }\end{array}$} & 4.1847 & 2 & 3.383 & 3.9113 & 4.3861 & 4.793 & $\begin{array}{l}\mathrm{F}(4.596)= \\
129.0765\end{array}$ \\
\hline & $\mathrm{n}=601$ & $\mathrm{n}=45$ & $\mathrm{n}=47$ & $\mathrm{n}=124$ & $\mathrm{n}=158$ & $\mathrm{n}=227$ & $\mathrm{p}=0.0000$ \\
\hline $\begin{array}{l}\text { Estoy satisfecho } \\
\text { con las actividades/ } \\
\text { servicios que ofrece } \\
\text { mi federación. }\end{array}$ & 3.8965 & 1.8372 & 2.9362 & 3.3871 & 4.0886 & 4.63 & $\begin{array}{c}F(4.594)= \\
130.8788\end{array}$ \\
\hline & $\mathrm{n}=599$ & $\mathrm{n}=43$ & $\mathrm{n}=47$ & $\mathrm{n}=124$ & $\mathrm{n}=158$ & $\mathrm{n}=227$ & $p=0.0000$ \\
\hline
\end{tabular}

Si llevamos a cabo un análisis de la varianza unidireccional tomando la felicidad como variable dependiente de la satisfacción del deportista, la hipótesis de independencia se rechaza, confirmándose la hipótesis plateada en este estudio. Además, el porcentaje de varianza explicada esta próximo al 50\%, siendo el coeficiente de determinación igual a 0.4629 .

De la misma forma, observando los resultados de ambas pruebas sobre las variables confianza y felicidad, nos encontramos con que ambas variables están relacionadas y, además, teniendo en cuenta los resultados observados en la tabla 4, existen diferencias significativas entre los diferentes grupos de la confianza y la felicidad:

\section{Tabla 4. Valores medios de los indicadores de confianza según el grado de felicidad de los federados}

\begin{tabular}{|c|c|c|c|c|c|c|c|}
\hline \multirow[b]{2}{*}{ Denominación } & \multirow[b]{2}{*}{$\begin{array}{c}\text { Total } \\
\text { muestra }\end{array}$} & \multicolumn{5}{|c|}{$\begin{array}{c}\text { Participar en las actividades de mi } \\
\text { federación me hace feliz }\end{array}$} & \multirow[b]{2}{*}{$\begin{array}{c}\text { F de } \\
\text { Snedecor }\end{array}$} \\
\hline & & 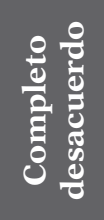 & 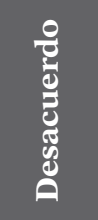 & 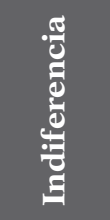 & 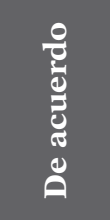 & 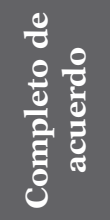 & \\
\hline \multirow[t]{2}{*}{$\begin{array}{l}\text { Mi federación se } \\
\text { ha comportado de } \\
\text { la manera que yo } \\
\text { esperaba en las ac- } \\
\text { tividades en las que } \\
\text { he participado. }\end{array}$} & 3.9185 & 1.9556 & 2.8936 & 3.4634 & 4.1582 & 4.5965 & $\begin{array}{c}\mathrm{F}(4.596)= \\
120.7371\end{array}$ \\
\hline & $\mathrm{n}=601$ & $\mathrm{n}=45$ & $\mathrm{n}=47$ & $\mathrm{n}=123$ & $\mathrm{n}=158$ & $\mathrm{n}=228$ & $\mathrm{p}=0.0000$ \\
\hline
\end{tabular}




\begin{tabular}{|c|c|c|c|c|c|c|c|}
\hline \multirow[b]{2}{*}{ Denominación } & \multirow[b]{2}{*}{$\begin{array}{c}\text { Total } \\
\text { muestra }\end{array}$} & \multicolumn{5}{|c|}{$\begin{array}{l}\text { Participar en las actividades de mi } \\
\text { federación me hace feliz }\end{array}$} & \multirow[b]{2}{*}{$\begin{array}{c}\text { F de } \\
\text { Snedecor }\end{array}$} \\
\hline & & 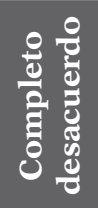 & 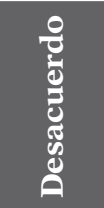 & 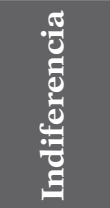 & 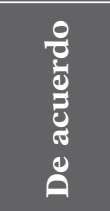 & 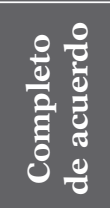 & \\
\hline \multirow[t]{2}{*}{$\begin{array}{l}\text { Mi federación tiene } \\
\text { un comportamiento } \\
\text { comprometido con } \\
\text { los federados a los } \\
\text { que prestan sus } \\
\text { actividades. }\end{array}$} & 3.9068 & 1.7111 & 2.8298 & 3.4758 & 4.1338 & 4.6404 & $\begin{array}{c}F(4.596)= \\
140.9658\end{array}$ \\
\hline & $\mathrm{n}=601$ & $\mathrm{n}=45$ & $\mathrm{n}=47$ & $\mathrm{n}=124$ & $\mathrm{n}=157$ & $\mathrm{n}=228$ & $\mathrm{p}=0.0000$ \\
\hline \multirow[t]{2}{*}{$\begin{array}{l}\text { Mi federación es } \\
\text { honesta con todos } \\
\text { federados. }\end{array}$} & 3.7663 & 1.5333 & 2.8511 & 3.377 & 3.8797 & 4.5286 & $\begin{array}{c}\mathrm{F}(4.594)= \\
113.3821\end{array}$ \\
\hline & $\mathrm{n}=599$ & $\mathrm{n}=45$ & $\mathrm{n}=47$ & $\mathrm{n}=122$ & $\mathrm{n}=158$ & $\mathrm{n}=227$ & $\mathrm{p}=0.0000$ \\
\hline \multirow[t]{2}{*}{$\begin{array}{l}\text { Mi federación se } \\
\text { preocupa de los } \\
\text { federados a los que } \\
\text { prestan sus activi- } \\
\text { dades. }\end{array}$} & 3.7629 & 1.5778 & 2.7826 & 3.2358 & 3.9557 & 4.5463 & $\begin{array}{c}\mathrm{F}(4.594)= \\
130.2304\end{array}$ \\
\hline & $\mathrm{n}=599$ & $\mathrm{n}=45$ & $\mathrm{n}=46$ & $\mathrm{n}=123$ & $\mathrm{n}=158$ & $\mathrm{n}=227$ & $\mathrm{p}=0.0000$ \\
\hline
\end{tabular}

Para la confianza, el análisis de la varianza también rechaza la hipótesis de independencia de esta variable respecto de la felicidad y además lo hace con una proporción de la varianza explicada del $42.85 \%$.

Por último, se analizan las variables del estudio que están relacionadas con las relaciones sociales. La primera variable es si se tiene relación personal con otros federados y la segunda si se tienen amigos dentro de la federación. Las pruebas de ji cuadrado nos confirman claramente que ambas variables están relacionadas tanto con la variable «Participar en las actividades de mi federación me hace feliz» como la variable «Los federados son felices cuando participan en las actividades de mi federación».

En la tabla 5, podemos observar el análisis de varianzas de la variable sobre la felicidad y la variable «tener trato personal con los federados», y podemos observar que las diferentes medias aritméticas de cada grupo van aumentando a medida que pasa de desacuerdo a acuerdo. A su vez, la prueba F de Snedecor con $\mathrm{p}=0.0000$ nos confirma que existen diferencias significativas en las medias aritméticas de los diferentes grupos. 
Tabla 5. Análisis unidireccional de la varianza sobre el grado de felicidad explicado por el trato personal con otros federados.

\begin{tabular}{|c|c|c|c|c|}
\hline Grupos & $\mathbf{N}^{\mathrm{o}}$ de casos & $\begin{array}{c}\text { Media } \\
\text { aritmética }\end{array}$ & $\begin{array}{c}\text { Desviación } \\
\text { estándar }\end{array}$ & $\begin{array}{c}\text { Suma de } \\
\text { cuadrados }\end{array}$ \\
\hline Total muestra & 599 & 3.7997 & 1.2339 & 911.9599 \\
\hline \multicolumn{5}{|c|}{ Categorias variable tratamiento } \\
\hline Completo desacuerdo & 34 & 2.4706 & 1.3982 & 66.4706 \\
\hline Desacuerdo & 44 & 2.9091 & 1.1245 & 55.6364 \\
\hline Indiferencia & 141 & 3.3404 & 1.0969 & 169.6596 \\
\hline De acuerdo & 142 & 3.7465 & 1.1097 & 174.8732 \\
\hline Completo de acuerdo & 238 & 4.458 & 0.955 & 217.0798 \\
\hline \multicolumn{4}{|r|}{ Suma: } & 683.7196 \\
\hline
\end{tabular}

En la tabla 6 observamos el análisis de varianza que compara el grado de felicidad del federado con la tenencia de amigos en la federación. Los resultados son muy similares a los de la tabla anterior, derivándose la principal diferencia en el coeficiente de determinación, que para la tabla 5 supone un 0.25 mientras que para la tabla 6 un 0.18 .

Tabla 6. Análisis unidireccional de la varianza sobre el grado de felicidad explicado por la tenencia de amigos en la federación

\begin{tabular}{|c|c|c|c|c|}
\hline Grupos & $\mathbf{N}^{o}$ de casos & $\begin{array}{c}\text { Media } \\
\text { aritmética }\end{array}$ & $\begin{array}{c}\text { Desviación } \\
\text { estándar }\end{array}$ & $\begin{array}{l}\text { Suma de } \\
\text { cuadrados }\end{array}$ \\
\hline Total muestra & 598 & 3.796 & 1.2303 & 905.1104 \\
\hline \multicolumn{5}{|c|}{ Categorías variable tratamiento } \\
\hline Completo desacuerdo & 18 & 2.1111 & 1.2862 & 29.7778 \\
\hline Desacuerdo & 16 & 3.1875 & 1.1842 & 22.4375 \\
\hline Indiferencia & 73 & 3.1644 & 1.1349 & 94.0274 \\
\hline De acuerdo & 127 & 3.3701 & 1.0781 & 147.6063 \\
\hline Completo de acuerdo & 364 & 4.1813 & 1.1094 & 448.033 \\
\hline \multicolumn{4}{|r|}{ Suma: } & 741.8819 \\
\hline
\end{tabular}

\section{Discusión y conclusiones}

Los resultados de este estudio son concluyentes, ya que todas las pruebas llevadas a cabo sobre la muestra obtenida conducen a la confirmación de las hipótesis planteadas en los apartados anteriores.

Al analizar la variable de la calidad percibida obtenemos que, tanto directa como indirectamente utilizando la satisfacción como variable mediadora, (H1) la cali- 
dad percibida influye en la felicidad del deportista federado español. Por lo tanto, en este caso el estudio empírico confirmaría los trabajos revisados en la teoría sobre calidad percibida y felicidad (Gong \& Yi, 2018; Sato et al., 2014; Theodorakis et al., 2015).

Los resultados también nos indican que la satisfacción y la felicidad tienen una fuerte relación y no solo eso, sino que la hipótesis (H2) planteada tras la revisión bibliográfica se confirma, teniendo una poderosa influencia sobre la felicidad del federado (H3), por lo tanto, coincidimos con los autores revisados (Dagger \& Sweeney, 2006; Gong \& Yi, 2018; Haller \& Hadler, 2006; Sweeney et al., 2015).

Respecto a la variable confianza, aunque el estudio de su influencia sobre la felicidad no es tan amplio en la literatura como el de las dos anteriores variables, existen varios autores que defienden que ambas variables tienen relación y que la felicidad se ve afectada por el grado de confianza en la organización (Barra et al., 2018; Bartolini \& Mikucka, 2017; Tokuda et al., 2010), y los resultados del estudio analizados coinciden con estos autores, dado que la hipótesis que se planteó sobre la influencia positiva de la confianza sobre la felicidad se confirma claramente (H4).

La última hipótesis planteada (H5), en la que se estudia la relación entre las relaciones sociales y la felicidad que aporta la actividad deportiva es rotunda, ya que todos los estadísticos confirman claramente la hipótesis de que las relaciones sociales influyen en la felicidad derivada del deporte. Por tanto, está claro que, para los federados en kárate españoles, tanto tener un trato personal con sus compañeros o tener lazos de amistad les aporta mayor felicidad. Esto se corresponde con lo revisado en la literatura, ya que para muchos autores tanto las relaciones sociales en general (Boudon, 2002; Gilbert, 2005; Haller \& Hadler, 2006) como los lazos de amistad (Jang et al., 2017) influyen en la felicidad de las personas.

De cara a los directivos de las federaciones, nuestras recomendaciones tras los resultados derivados de esta investigación son las de maximizar en lo posible la calidad de sus servicios ya que esta variable tiene una mayor explicación que el resto de las variables, además de influir no solo en la felicidad sino en la satisfacción del federado (Gong \& Yi, 2018). Todo ello sin olvidarse de la importancia que supone la variable confianza, ya que la existencia de confianza por parte del federado ayuda a mantener la felicidad en momentos en los que no se pueda tener el mejor servicio o los mejores resultados (Barra et al., 2018; Tokuda et al., 2010). Y por supuesto sin olvidarnos de que fortalecer las relaciones sociales entre todas las personas que participan de alguna forma en la federación, motivando la amistad y el compañerismo será otra de las piezas claves para mantener a los federados felices (Haller \& Hadler, 2006).

\section{Apoyos y soporte financiero de la investigación}

Entidad: Universidad Politécnica Salesiana de Ecuador

País: Ecuador

Ciudad: Cuenca

Entidad: Federación Regional de Kárate

País: España

Ciudad: Madrid 


\section{Referencias}

Aksoy, L., Keiningham, T. L., Buoye, A., Larivière, B., Williams, L., \& Wilson, I. (2015). Does loyalty span domains? Examining the relationship between consumer loyalty, other loyalties and happiness. Journal of Business Research, 68(12), 2464-2476. https://doi.org/10.1016/j. jbusres.2015.06.033

Anderson, E. W., \& Mansi, S. A. (2009). Does customer satisfaction matter to investors? Findings from the bond market. Journal of Marketing Research, 46(5), 703-714. https://doi.org/10.1509/ jmkr.46.5.703

Argyle, M. (1994). The Psychology of Happiness. London, UK: Routledge. https://doi. org/10.4324/9781315812212

Barra, C., Pressgrove, G., \& Torres, E. (2018). Trust and commitment in the formation of donor loyalty. The Service Industries Journal, 38(5-6), 360-377. https://doi.org/10.1080/02642069.201 7.1405937

Barriopedro, E. N., Ripoll, R. R., \& Tello, E. A. (2018a). Análisis de la situación actual de la felicidad corporativa de los millennials en España, desde el barómetro del Centro de Investigaciones Sociológicas. Revista Académica ECO 18, 1-22.

Barriopedro, E. N., Ripoll, R. R., \& Tello, E. A. (2018b). El impacto del crecimiento económico y empresarial en la felicidad social de la población española. En Hernández G. de Velazco, J. J., y Romero Marín, L. C., (Eds.), Perspectivas en Ciencias Jurídicas y Políticas sobre la participación ciudadana, paz, bienestar y felicidad social siglo XXI (pp. 190-210). Barranquilla, Colombia: Educosta SAS.

Bartolini, S., Mikucka, M., \& Sarracino, F. (2017). Money, Trust and Happiness in Transition Countries: Evidence from Time Series. Social Indicators Research, 130(1), 87-106. https://doi. org/10.1007/s11205-015-1130-3

Bolifa, F., Cuesta Valiño, P., Núñez-Barriopedro, E., \& Penelas Leguía, A. (2018). Los adolescentes musulmanes frente a las oportunidades y las amenazas de la red. Revista Internacional de Investigación en Comunicación aDResearch ESIC, 18(18), 60-79. https://doi.org/10.7263/adresic-018-04

Boudon, R. (2002). Declin de la morale? Declin des valeurs?. Quebec, Canadá: Editions Nota Bene. https://doi.org/10.7202/007464ar

Campillo Álvarez, J. E. (2007). El mono obeso: la evolución humana y las enfermedades de la opulencia: diabetes, hipertensión, arteriosclerosis. Barcelona, España: Crítica.

Consejo Superior de Deportes. (2019). Deporte de Alto Nivel y Alto Rendimiento, 01 de mayo de 2019, Recuperado de https://bit.ly/30rtnzM [Fecha de consulta: 01 de mayo de 2019].

Cuesta Valiño, P., Gutiérrez Rodríguez, P., \& Núñez Barriopedro, E. (2019). The impact of corporate social responsibility on customer loyalty in hypermarkets : A new socially responsible strategy. Corporate Social Responsability and Environmental Management, 26(4), pp. 761-769. https://doi.org/10.1002/csr.1718

Dagger, T. S., \& Sweeney, J. C. (2006). The Effect of Service Evaluations on Behavioral Intentions and Quality of Life. Journal of Service Research, 9(1), 3-18. https://doi. org/10.1177/1094670506289528

Demir, M., \& Urberg, K. A. (2004). Friendship and Adjustment among Adolescents. Journal of Experimental Child Psychology, 88(1), 68-82. https://doi.org/10.1016/j.jecp.2004.02.006

Demir, M., \& Weitekamp, L. A. (2007). I am so happy cause today I found my friend: friendship and personality as predictors of happiness. Journal of Happiness Studies, 8(2), 181-211. https://doi.org/10.1007/s10902-006-9012-7

Easterlin, R. A. (2001). Income and Happiness: Towards a Unified Theory. The Economic Journal, 111(473), 465-484. https://doi.org/10.1111/1468-0297.00646 
Eaton J., Gwinner K., Larson B., \& Swanson S. (2015) The Role of Spectators' Attitudes Toward Commercialization on the Effectiveness of Corporate Sponsorhip of Sporting Events. En Moore, M., Moore, R. (Eds.), New Meanings for Marketing in a New Millennium. Developments in Marketing Science: Proceedings of the Academy of Marketing Science (pp 263-263). California, Estados Unidos: Springer Cham. https://doi.org/10.1007/978-3-319-11927-4_87

Gallarza, M. G., Arteaga, F., Del Chiappa, G., Gil-Saura, I., \& Holbrook, M. B. (2017). A multidimensional service-value scale based on Holbrook's typology of customer value: bridging the gap between the concept and its measurement. Journal of Service Management, 28(4), 724762. https://doi.org/10.1108/JOSM-06-2016-0166

Gilbert, D. T. (2005). Stumbling Happiness. New York, Estados Unidos: Vintage Books.

Gong, T., \& Yi, Y. (2018). The effect of service quality on customer satisfaction, loyalty, and happiness in five Asian countries. Psychology $\mathcal{E}$ Marketing, 35(6), 427-442. https://doi.org/10.1002/ mar.21096

Haller, M. A. X., \& Hadler, M. (2006). How social relations and structures can produce happiness and unhappiness: an international comparative analysis. Social Indicators Research, 75(2), 169-216. https://doi.org/10.1007/s11205-004-6297-y

Hallmann, K., Breuer, C., \& Ku, B. (2013). Happiness, pride and elite sporting success: What population segments gain most from national athletic achievements? Sport Management Review, 16(2), 226-235. https://doi.org/10.1016/j.smr.2012.07.001

Hennig-Thurau, T., Gwinner, K. P., \& Gremler, D. D. (2002). Understanding relationship marketing outcomes: An integration of relational benefits and relationship quality. Journal of Service Research, 4(3), 230-247. https://doi.org/10.1177\%2F1094670502004003006

Hennig-Thurau, T., Langer, M. F., \& Hansen, U. (2001). Modeling and managing student loyalty: An approach based on the concept of relationship quality. Journal of Service Research, 3(4), 331-344. https://doi.org/10.1177\%2F109467050134006

Huang, H., \& Humphreys, B. R. (2012). Sports participation and happiness: Evidence from US microdata. Journal of Economic Psychology, 33(4), 776-793. https://doi.org/10.1016/j. joep.2012.02.007

Hur, Y., Ko, Y. J., \& Valacich, J. (2011). A Structural Model of the Relationships Between Sport Website Quality, E-Satisfaction, and E-Loyalty. Journal of Sport Management, 25(5), 458-473. https://doi.org/10.1123/jsm.25.5.458

Jang, W., Ko, Y. J., Wann, D. L., \& Kim, D. (2017). Does Spectatorship Increase Happiness? The Energy Perspective. Journal of Sport Management, 31(4), 333-344. https://doi.org/10.1123/ jsm.2016-0113

Javalgi, R. R. G., \& Moberg, C. R. (1997). Service loyalty: implications for service providers. Journal of Services Marketing, 11(3), 165-179. https://doi.org/10.1108/08876049710168663

Johnson, D., \& Grayson, K. (2005). Cognitive and affective trust in service relationships. Journal of Business Research, 58(4), 500-507. https://doi.org/10.1016/S0148-2963(03)00140-1

Khan, S., \& Hussain, M. (2013). Determinants of Consumer Happiness and Its Role in Customer Loyalty. International Review of Management and Business Research, 2(1), 11-19. https://dx.doi. org/10.2139/ssrn.2269677

Nelson, S. K., Della Porta, M. D., Jacobs Bao, K., Lee, H. C., Choi, I., \& Lyubomirsky, S. (2015). 'It's up to you': Experimentally manipulated autonomy support for prosocial behavior improves well-being in two cultures over six weeks. The Journal of Positive Psychology, 10(5), 463-476. https://doi.org/10.1080/17439760.2014.983959

Nicolao, L., Irwin, J. R., \& Goodman, J. K. (2009). Happiness for sale: Do experiential purchases make consumers happier than material purchases? Journal of Consumer Research, 36(2), 188198. https://doi.org/10.1086/597049

Núñez-Barriopedro, E., Ruiz-López, J., \& Ravina Ripoll, R. (2018). La creatividad en el sector turístico americano y europeo: Caso Croacia. Retos: Revista de Ciencias de la Administración y Economía, 8(15), 83-99. http://dx.doi.org/10.17163/ret.n15.2018.06 
Núñez-Barriopedro, E., Ravina Ripoll, R. \& Tobar Pesántez, L.B. (2019). La creatividad: un capital intangible como generador de calidad competitiva en el sector publicitario español. Academy of Accounting and Financial Studies Journal, 23(2).

Ravina-Ripoll, R., Núñez-Barriopedro, E., Galiano-Coronil, A., \& Tobar-Pesántez, L. B. (2019). Towards a happy, creative and social higher education institution: the case of non-profit marketing and business creation subjects at the University of Cadiz. Journal of Entrepreneurship Education, 22(1).

Romero-Rodriguez, L.M. \& Castillo-Abdul, B (2019). Comunicación para la motivación. Claves de la asertividad y del trabajo en equipo en las organizaciones. En Ravina Ripoll, R., Tobar Pesántez, L.B., y Núñez Barriopedro, E. (Eds.) Happiness management and creativity in the XXI century: Intangible capitals as a source of innovation, competitiveness and sustainable development (pp. 41-54) Granada: España: Comares.

Sato, M., Jordan, J. S., \& Funk, D. C. (2014). The role of physically active leisure for enhancing quality of life. Leisure Sciences, 36(3), 293-313. https://doi.org/10.1080/01490400.2014.886912

Seligman, M. E., \& Csikszentmihalyi, M. (2014). Positive psychology: An introduction. In Flow and the foundations of positive psychology (pp. 279-298). Dordrecht, Países Bajos: Springer. https:// doi.org/10.1007/978-94-017-9088-8

Smith, A., \& Stewart, B. (2010). The special features of sport: A critical revisit. Sport Management Review, 13(1), 1-13. https://doi.org/10.1016/j.smr.2009.07.002

Sweeney, J. C., Danaher, T. S., \& Mccoll-kennedy, J. R. (2015). Customer Effort in Value Cocreation Activities: Improving Quality of Life and Behavioral Intentions of Health Care Customers. Journal of Service Research, 18(3), 318-335. https://doi.org/10.1177/1094670515572128

Theodorakis, N. D., Kaplanidou, K. (KIKI), \& Karabaxoglou, I. (2015). Effect of Event Service Quality and Satisfaction on Happiness Among Runners of a Recurring Sport Event. Leisure Sciences, 37(1), 87-107. https://doi.org/10.1080/01490400.2014.938846

Tokuda, Y., Fujii, S., \& Inoguchi, T. (2010). Individual and Country-Level Effects of Social Trust on Happiness: The Asia Barometer Survey. Journal of Applied Social Psychology, 40(10), 25742593. https://doi.org/10.1007/978-981-10-2305-7_8

Wemmer, F., \& Koenigstorfer, J. (2016). Open Innovation in Nonprofit Sports Clubs. International Journal of Voluntary and Nonprofit Organizations, 27(4), 1923-1949. https://doi.org/10.1007/ s11266-015-9571-5 